\title{
Hardness Variation of Microstructural Heterogeneities in Directed Energy Deposited 304L Stainless Steel
}

\author{
Heidy Vega ${ }^{1}$, Thale R. Smith ${ }^{1}$, Chris San Marchi ${ }^{1}$, Raymond W. Friddle ${ }^{1}$ and Joshua D. Sugar ${ }^{1}$ \\ 1. Sandia National Laboratories, Livermore, CA, USA. \\ * Corresponding author: heivega@sandia.gov
}

The rapid solidification that occurs during additive manufacturing processes results in a cellular structure with compositional variation across the cell boundaries. It has been shown that the dislocation interaction with the cell boundaries defined by this compositional microsegregation can impact the yield strength of the material [1]. While the Vicker's Hardness method can be used to measure the hardness of a bulk material, this technique cannot be used to measure the hardness of individual cell boundaries because the required indentation diagonal, $17 \mu \mathrm{m}$ [2], is much larger than the 1-2 um solidification cells. Nanoindentation, however, uses indent loads between $1 \mathrm{uN}$ to $100 \mathrm{mN}$ to measure localized hardness of a material within a small area. The ability to measure hardness within a small volume of the material makes it a promising technique for isolating the mechanical properties of the distinct microstructural features in these materials that occur at the micron and submicron length scale.

The goal of this study is to combine nanoindentation and microscopy techniques to understand the contribution of distinct microstructural features to the overall strength of additively manufactured 304L stainless steel. With the small length scale of the nano-indenter, it is possible to individually probe the hardness of features such as the cell boundaries mentioned above, the retained ferrite, grain boundaries, and oxide inclusions. The overall strength of this material can then be understood by considering the contributions of each of these features and their interaction with each other. Figure 1a) shows a typical etched optical micrograph of DED 304L where the individual build layers and melt pools are visible and outlined in red. Figure 1b) is a higher magnification image where the solidification cells and ferrite are now visible. Figure 1c) shows an SEM micrograph of an array of indents, and after indentation, their vicinity to the various microstructural features can be determined with SEM. Figure 2 a) and b) show a corresponding SEM micrograph and EDS map ( $\mathrm{Cr}$ in green, $\mathrm{Ni}$ in red) that demonstrates qualitatively the Cr-enrichment at the cell boundaries. Figure 3 a) and b) show typical scanning probe microscopy images after indentation, and c) shows the corresponding load-displacement curves that demonstrate the material response depends on the details of the microstructural environment being interrogated. Combined nanoindentation and microscopy measurements like these are important tools that provide insight into microstructure-property relationships in these complex materials [3].

References:

[1] TR Smith et al., Acta Materialia, 164 (2019), p. 728.

[2] ASTM E384-11, Standard Test Method for Knoop and Vickers Hardness of Materials, ASTM International, West Conshohocken, PA (2011) www.astm.org

[3] Sandia National Laboratories is a multimission laboratory managed and operated by National Technology and Engineering Solutions of Sandia LLC, a wholly owned subsidiary of Honeywell International Inc., for the U.S. Department of Energy's National Nuclear Security Administration under contract DE-NA0003525. 
a)

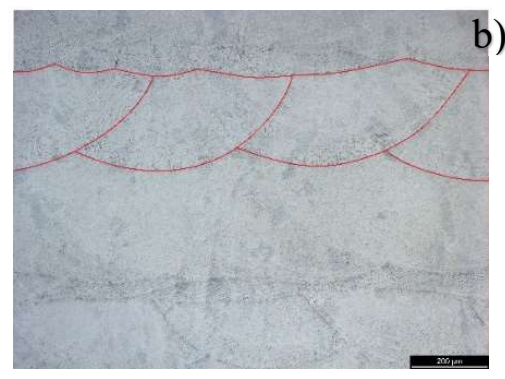

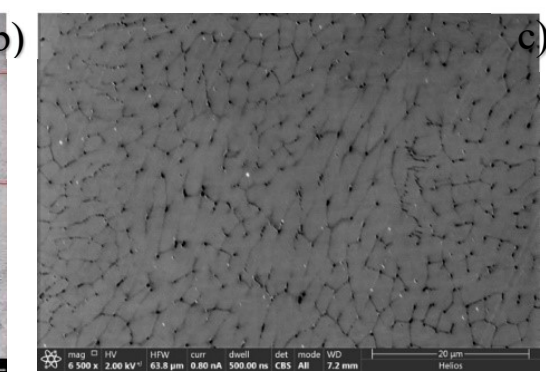

Figure 1. (a) Optical image of a light $10 \% \mathrm{NaOH}$ etched 304L stainless steel. The red lines highlight the locations of the melt pools from each laser pass. (b) SEM micrograph of solidification cells within the tracks. (c) SEM micrograph showing an array of indentations that probe various microstructural features (highlighted with red circles).
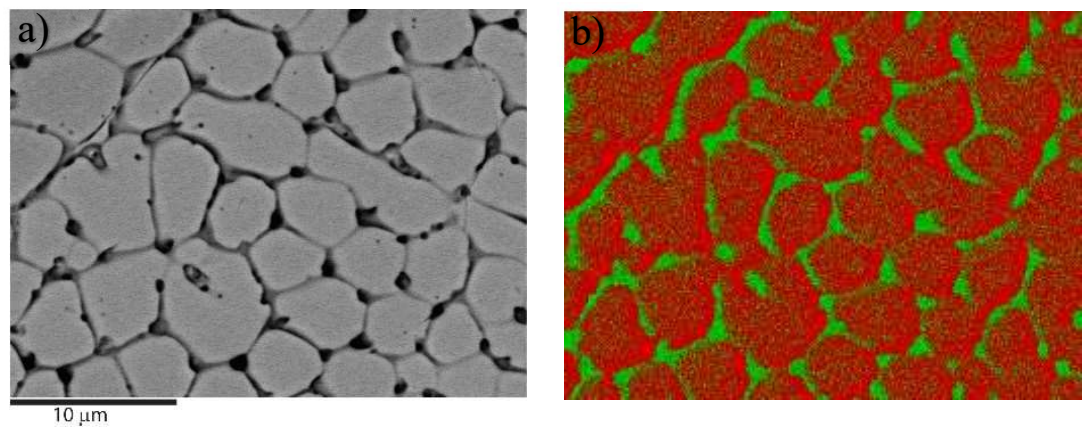

\section{Cr-rich}

Ni-rich

Figure 2. (a) SEM micrograph and (b) corresponding EDS map ( $\mathrm{Cr}$ in green, $\mathrm{Ni}$ in red) showing compositional micro-segregation of DED 304L stainless steel.
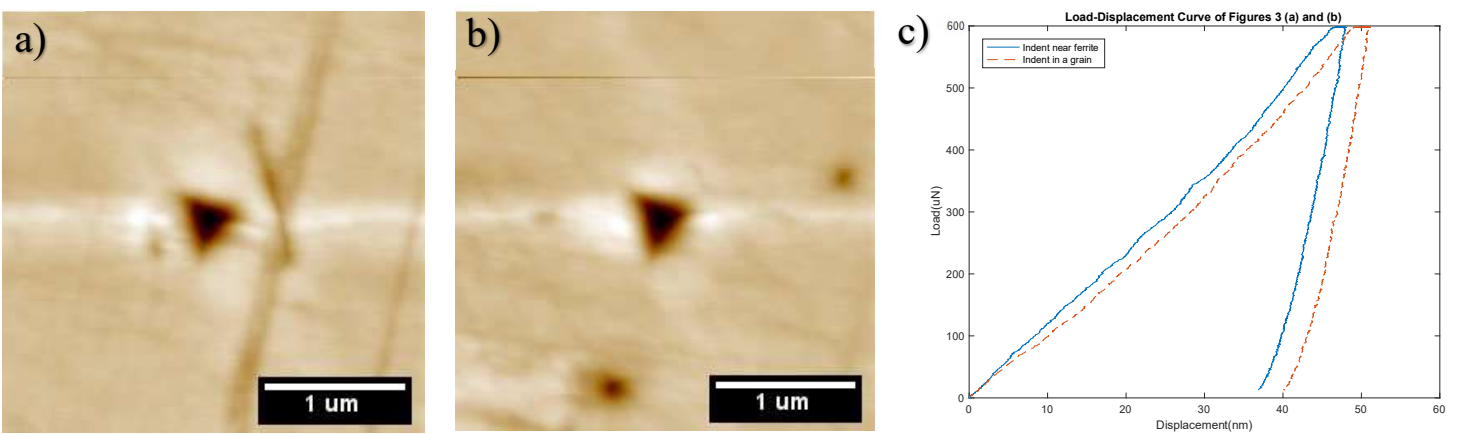

Figure 3. Scanning probe microscopy image of an indent (a) near ferrite, and (b), in the middle of a grain boundary as determined by comparison with Figure 1 (c). Load-Displacement curves (c) for the indents shown in (a) and (b) near ferrite and near a grain. 